\title{
The combination of camrelizumab and apatinib obtained ongoing partial remission for a patient with osimertinib-resistant non-small cell lung cancer: case report
}

\author{
Xiufeng Cong, Jun Chen, Wei Zheng \\ Department of Oncology, Shengjing Hospital of China Medical University, Shenyang, China \\ Correspondence to: Wei Zheng. Department of Oncology, Shengjing Hospital of China Medical University, Shenyang, China. Email: zhengw67@163.com.
}

\begin{abstract}
Extensive clinical studies have indicated that the epidermal growth factor receptor (EGFR)tyrosine kinase inhibitors (TKIs) can significantly improve the survival rate of patients with EGFRmutation-positive malignancies. However, acquired resistance to the third-generation EGFR-TKI osimertinib is an intractable obstacle for many clinical oncologists. The resistance mechanism of osimertinib is very complicated, and the individual treatment varies greatly. We present the case of a 76-year-old woman with advanced non-small cell lung cancer (NSCLC) with EGFR L858R mutation, as well as multiple lung metastases and multiple liver metastases. The patient's lung lesions progressed after almost 2 years of treatment with Osimertinib. Due to poor physical condition, she could not tolerate chemotherapy or invasive examination. A next-generation sequencing (NGS) panel of a plasma sample showed missense mutations of KRAS (G12S), MET (D1028Y), AR (S697P), LRP1B (S2662C) with allelic frequencies of 0.6\%, 0.5\%, 0.2\%, $0.2 \%$, respectively), 2 nonsense mutations [ZNF521 $\left(E 307^{*}\right), \operatorname{MET}\left(Q 42^{*}\right)$, with allelic frequencies of $0.5 \%$, $0.3 \%$, respectively], and a splicing mutation in FAT1 (c.3266-1G>C) with an allelic frequency of $0.5 \%$. After treatment with camrelizumab (200 mg fortnightly) combined with small dose of apatinib (125 mg qd), the patient's lung lesions were successfully overcome with significant reduction and necrosis formation. And the patient's symptoms were significantly relieved and was well tolerated. To our knowledge, this is the first report on the successful treatment of such patients. It indicated a promising treatment option in the clinic to the NSCLC with osimertinib resistance.
\end{abstract}

Keywords: Epidermal growth factor receptor (EGFR); camrelizumab; apatinib; non-small cell lung cancer (NSCLC); case report

Submitted Nov 06, 2019. Accepted for publication Sep 02, 2020.

doi: 10.21037/apm-19-462

View this article at: http://dx.doi.org/10.21037/apm-19-462

\section{Introduction}

The first line treatment for a patient with advanced nonsmall cell lung cancer (NSCLC) with epidermal growth factor receptor (EGFR) gene mutations has been the EGFR tyrosine kinase inhibitors (TKIs). Osimertinib is an EGFR-TKI that has been used worldwide to treat EGFRmutated NSCLCs with TKI resistance mediated by the EGFR T790M mutation (1). However, clinical practice has found that acquired resistance to osimertinib develops frequently during treatment, and resistance to osimertinib has become a major clinical challenge that remains poorly understood (2).

Therefore, investigations have focused on novel combination therapies, which have gradually revealed that combination therapies might become promising options. The previous IMpower150 trial has shown that EGFRpositive patients with TKI resistance can be effectively treated by chemotherapy combined with antiangiogenic therapy and immunotherapy (3). Camrelizumab (SHR1210 ) is a novel anti-programmed cell death-1 (antiPD-1) antibody that was developed in China and has 
been proven to be effective for a variety of cancers (4). Apatinib is a new type of small-molecule TKI that was also developed in China. It was found to bind selectively to vascular endothelial growth factor receptor 2 (VEGFR-2), which leads to decreased VEGF-mediated endothelial cell migration, proliferation, and tumor microvascular density $(5,6)$.

Here we report a patient with primary lung adenocarcinoma and multiple hepatic metastases that became resistant to first- and third-generation TKIs during treatment. She could not tolerate chemotherapy during the poor performance status. After the combination treatment of camrelizumab (SHR-1210) plus apatinib, the patient's clinical signs and symptoms and condition rapidly improved. This is the first case report that successfully achieved partial remission to a osimertinib-resistant NSCLC patient using this therapy. Our patient's outcome indicates that camrelizumab (SHR-1210) plus apatinib could be a promising option for the clinical treatment of patients with EGFR-TKI-resistant NSCLCs. We present the following case in accordance with the CARE Reporting Checklist (available at http://dx.doi.org/10.21037/apm-19-462).

\section{Case presentation}

The patient was a 76-year-old woman with a negative history of smoking and a negative family history of genetic disease. In July 2015, her physical examination revealed lung lesions in with no clinical symptoms. Then she underwent ${ }^{18} \mathrm{~F}$-fluorodeoxyglucose (FDG) positron emission tomography-computed tomography (PET-CT) and was diagnosed with stage IV NSCLC and multiple nodules of liver metastases based on the 7th Edition of the American Joint Committee on Cancer (7). The PETCT results showed the following: elevated FDG uptake by a mass in the posterior segment of the left upper lung, which strongly suggested malignant lung cancer; groundglass densities and nodules with elevated FDG uptake in the basal segment of the right lower lobe, both findings strongly suggestive lung cancer; and locally elevated FDG uptake in liver nodules near the fourth posterior rib on the right side, suggestive of metastases (Figure 1). The patient underwent a percutaneous lung biopsy of the left upper lobe in July 2015. The histopathological results confirmed a primary adenocarcinoma that was a moderately-highly differentiated EGFR-mutated lung adenocarcinoma with an L858R mutation in exon 21.

\section{Therapeutic interventions}

The patient was started on gefitinib (250 $\mathrm{mg}$ qd orally) in August 2015. A partial response (PR, modified RESIST1.1 Criteria) was achieved after 1 month of treatment. The lung lesions were evaluated as stable disease (SD, modified RESIST1.1 Criteria) and the liver lesions were evaluated as PR (maximum baseline diameter of lesion in S3 was reduced from $1.7 \mathrm{~cm}$ to $8 \mathrm{~mm}$ and the lesion in $\mathrm{S} 7$ could not be seen clearly.) The PR (modified RESIST1.1 Criteria) was maintained until August 2017, with a progression-freesurvival (PFS) time of 25 months for the patient's first-line therapy.

On the last day of August 2017, the patient underwent PET-CT, which showed the following: the mass with elevated FDG uptake in the left upper lobe on previous PET-CT had become slightly reduced in size, but the FDG uptake was increased [maximum standardized uptake value $\left(\mathrm{SUV}_{\text {max }}\right)$ had increased from 9.2 to 12.6]; the $\mathrm{S} 7$ hepatic nodules were larger $(2.5 \mathrm{~cm})$, and FDG uptake was increased over previous PET-CT $\left(\mathrm{SUV}_{\max }\right.$ increased from 3.8 to 7.6); and the $S 3$ hepatic nodules were smaller than previously $(1.2 \mathrm{~cm})$, and the FDG uptake value was similar to that of normal liver parenchyma.

In September 2017, the patient underwent percutaneous liver biopsy, which was confirmed to metastatic adenocarcinoma. Gene sequencing studies were positive for EGFR mutations (L858R in exon 21 and T790M in exon 20). The patient was started on oral osimertinib (80 $\mathrm{mg} \mathrm{qd}$ ); and 1 month later, CT revealed findings indicating a PR (modified RESIST1.1 Criteria), with her lung lesions considered to be SD, her S7 liver lesion reduced to $1.3 \mathrm{~cm}$, and her $\mathrm{S} 3$ liver lesion barely visible.

The patient's PFS on second-line therapy was 23 months. In August 2019, the patient started coughing, had breathing problems, and gradually worsened. Her Eastern Cooperative Oncology Group (ECOG) performance status was worsened to 3 . Chest $\mathrm{CT}$ revealed marked progression of her bilateral lung lesions. The S7 liver lesion was unchanged $(1.0 \mathrm{~cm})$. Because of the patient's poor physical condition and severe breathing difficulties, she was unable to tolerate another lung biopsy. A next-generation sequencing (NGS) panel of a plasma sample showed 7 somatic mutations, including 4 missense mutations [KRAS (G12S), MET (D1028Y), AR (S697P), LRP1B (S2662C) with allelic frequencies of $0.6 \%$, $0.5 \%, 0.2 \%, 0.2 \%$, respectively], 2 nonsense mutations [ZNF521 (E307*), MET (Q42*), with allelic frequencies 


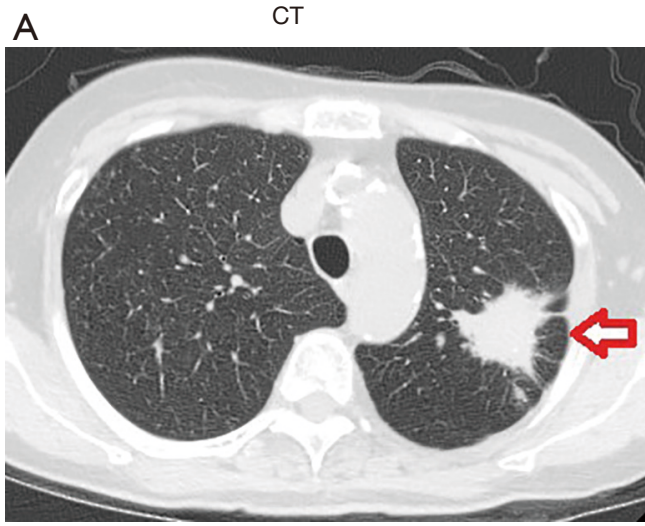

B
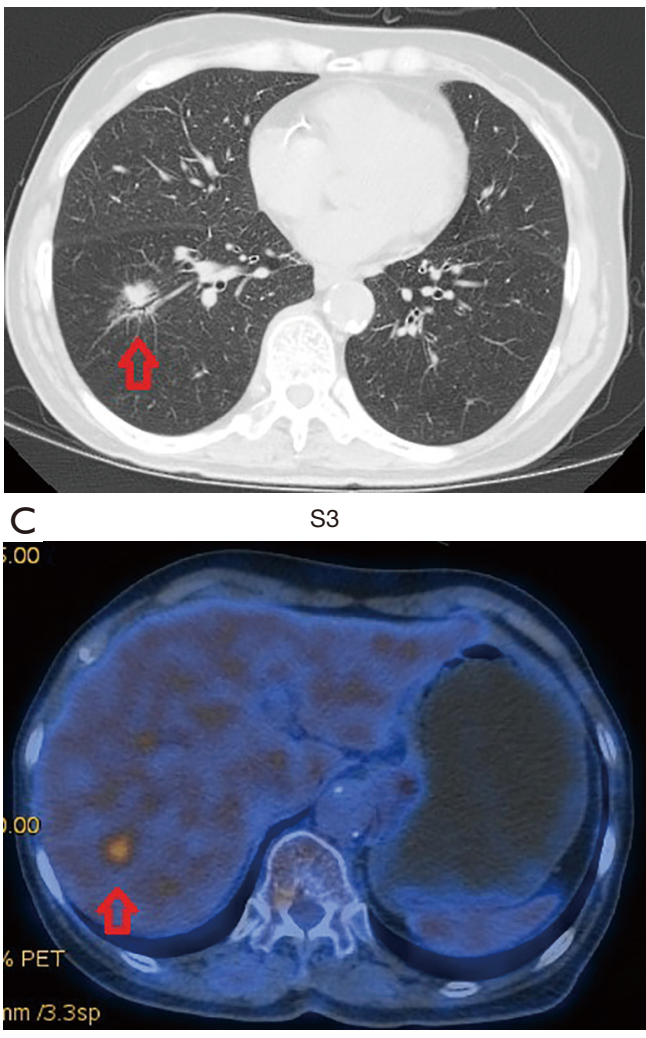

PET-CT fused image

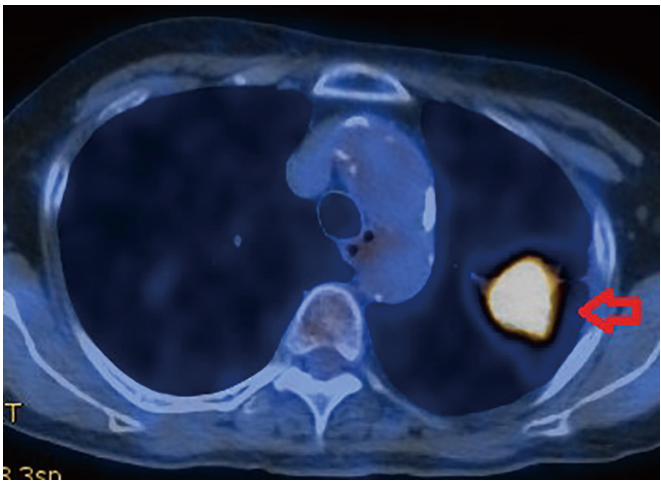

PET-CT fused image

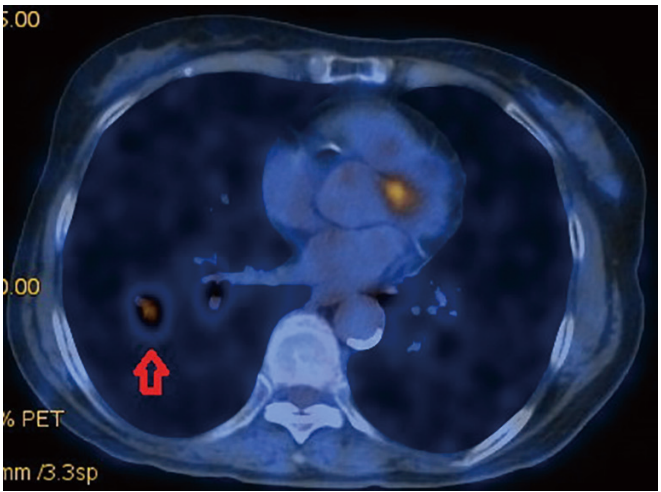

S7

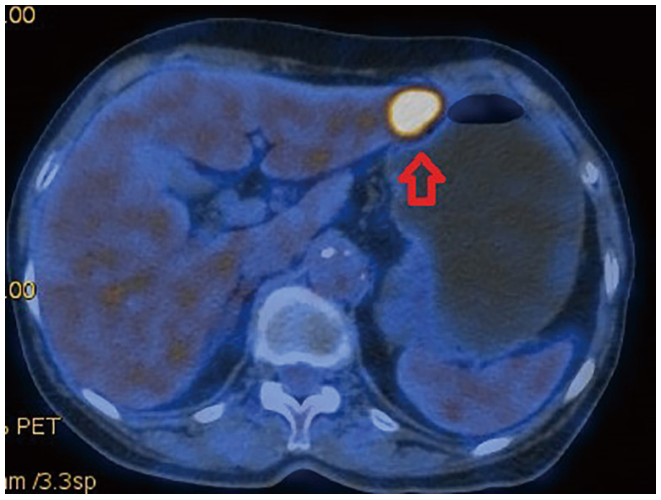

Figure 1 PET-CT imaging at baseline in July 2015. (A) The lesion in the upper lobe of the left lung; (B) the lesion in the lower lobe of right lung; (C) PEG-CT imaging of liver metastasis in S3 and S7. The arrows indicate tumors.

of $0.5 \%, 0.3 \%$, respectively], and a splicing mutation in FAT1 (c.3266-1G>C) with an allelic frequency of $0.5 \%$ (Table 1). Since genetic testing of the plasma specimen did not reveal an EGFR mutation, and the patient's pulmonary lesions had progressed and her ECOG score was poor, she was administered camrelizumab (SHR-1210, $200 \mathrm{mg}$ fortnightly) and apatinib (125 $\mathrm{mg}$ qd) during a 4-week cycle.

\section{Follow-up and outcomes}

After one cycle of treatment, a follow-up chest CT revealed marked reduction in the bilateral pulmonary lesions and signs of necrosis (Figure 2). The hepatic lesions were found to be unchanged (SD). The patient's breathing problems were markedly reduced, and her ECOG performance status improved from 3 to 1 . The patient is still receiving treatment and to date we have not observed treatment- 
Table 1 The gene mutation profiling results of plasma testing after progression of osimertinib

\begin{tabular}{|c|c|c|c|c|}
\hline Gene & Mutation type & DNA change & Amino acid change & Mutation frequency (\%) \\
\hline FAT1 & Splicing & c. $3266-1 \mathrm{G}>\mathrm{C}$ & - & 0.5 \\
\hline MET & Missense & c. $3082 \mathrm{G}>\mathrm{T}$ & p.D1028Y & 0.5 \\
\hline ZNF521 & Nonsense & c. $919 \mathrm{G}>\mathrm{T}$ & p.E307* & 0.5 \\
\hline$A R$ & Missense & c. $2089 T>C$ & p.S697P & 0.2 \\
\hline$L R P 1 B$ & Missense & c. $7985 C>G$ & p.S2662C & 0.2 \\
\hline
\end{tabular}
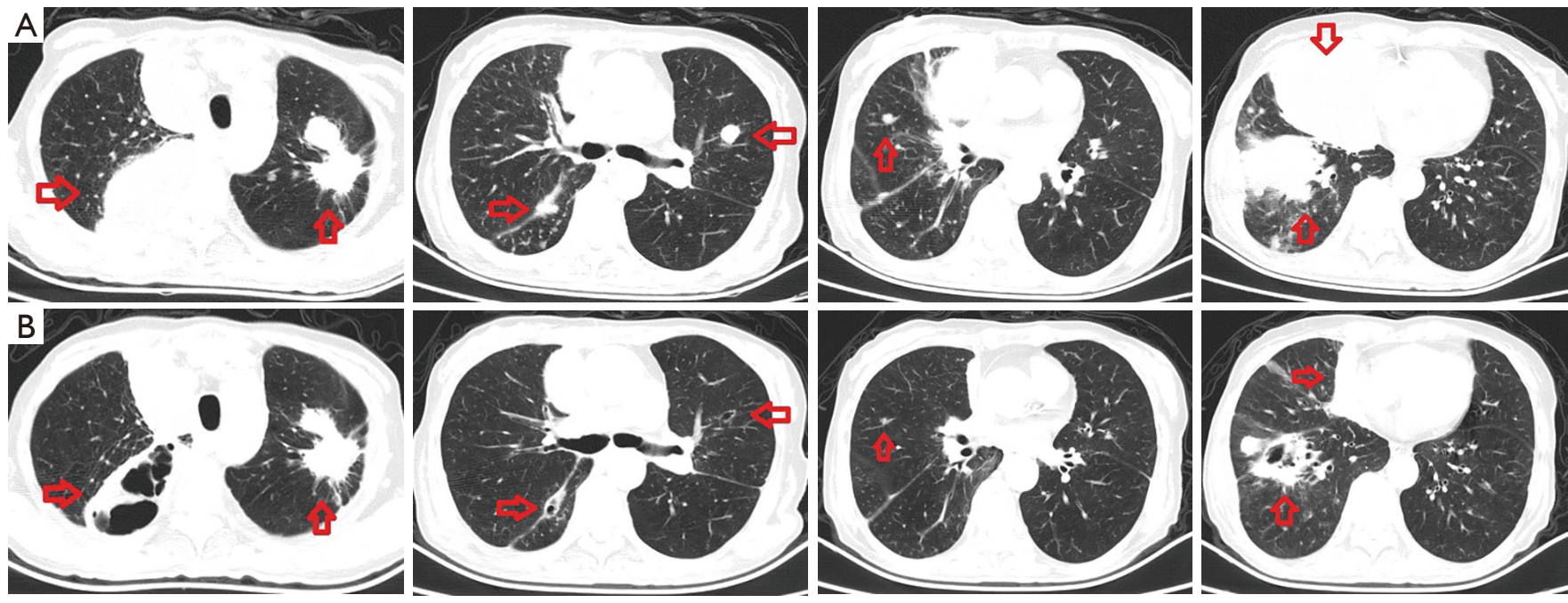

Figure 2 The treatment efficacy of camrelizumab and apatinib combination. (A) CT imaging of lung tissue before camrelizumab and apatinib treatment (August 7, 2019); (B) CT imaging of lung tissue after camrelizumab and apatinib treatment (September 16, 2019). The arrows indicate tumors.

related side effects. Followed up to March 2020, the patient's lung lesions are still partial remission and her quality of life is good (Figure 3).

All procedures performed in studies involving human participants were in accordance with the ethical standards of the institutional and/or national research committee(s) and with the Helsinki Declaration (as revised in 2013). Written informed consent was obtained from the patient for publication of this manuscript and any accompanying images.

\section{Discussion}

EGFR-TKI agents have obtained remarkable survival benefits for patients with EGFR-positive NSCLC; however, resistance to TKI agents has become a major clinical problem. Unfortunately, acquired resistance is inevitable after long-term treatment. To date, the drug resistance mechanism has not been completely clarified. The theoretical explanations with the greatest acceptance include acquired EGFR mutations (e.g., T790M), activation of bypass signaling pathways, such as mesenchymal epithelial transition (MET) factor and human epidermal growth factor receptor-2 (HER2), and small-cell transformation. About $60 \%$ of patients have the exon 20 resistance mutation T790M (8). The AURA3 study (clinical trials.gov number, NCT02151981) found that osimertinib effectively treated patients with EGFR-T790M-positive NSCLC, who obtained significantly improved progression-free survival (PFS) (1). 


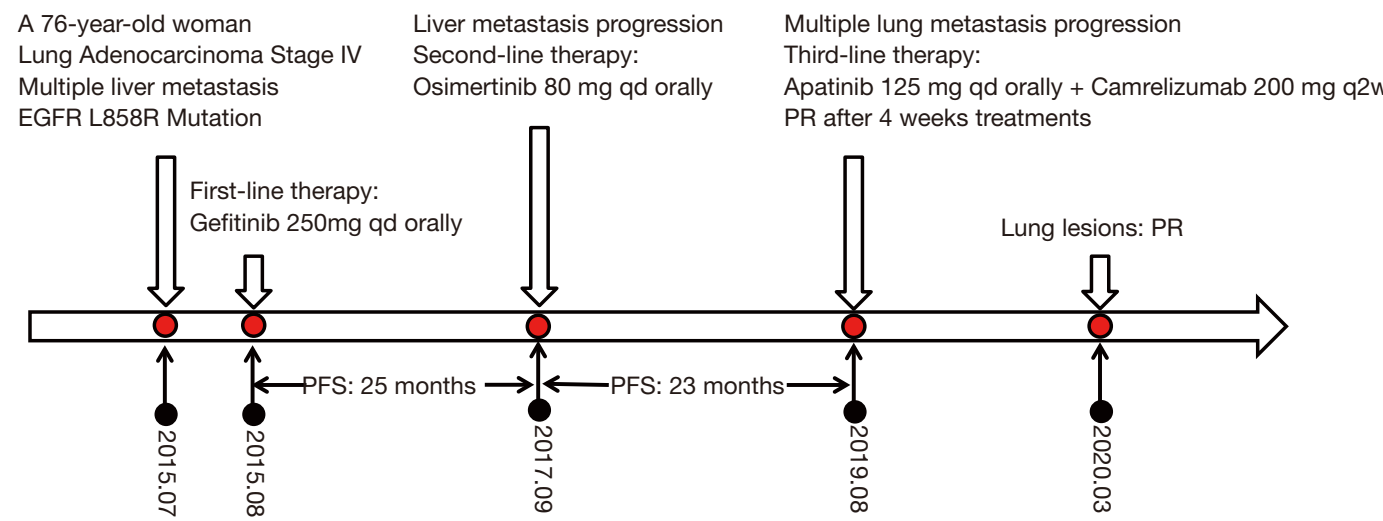

Figure 3 Timeline of the patient's therapy. EGFR, epidermal growth factor receptor; PFS, progression free survival; PR, partial response; qd, once a day; q2w, every 2 weeks.

With the widespread use of osimertinib, the mechanisms of resistance to it and treatment strategy after drug resistance have been studied in depth. EGFR-dependent and $E G F R$-independent mechanisms have been reported in both preclinical and clinical settings. EGFR-dependent mechanisms include the development of the EGFR-C797S mutation, which is one of the most commonly reported mutations conferring resistance. The EGFR-independent mechanisms mainly include MET and HER2 amplification, the fibroblast growth factor receptor 1 (FGFR 1), the PIK3CA mutation, small-cell transformation, and activation of the RAS-MAPK pathway (9).

The acquired resistance to osimertinib mediated by loss of the T790M mutation is associated with early resistance. The clinical data have indicated that advanced NSCLCs develop heterogeneous resistance mechanisms, and investigations of therapeutic strategies that can overcome multiple mechanisms of drug resistance are urgently needed (2).

The IMpower150 trial (ClinicalTrials.gov, number NCT0236614) was a randomized, open-label phase 3 study performed at 240 academic medical centers and community oncology practices in 26 countries. Efficacy was assessed in key subgroups within the intention-to-treat population, including NSCLC patients with EGFR mutations previously treated with 1 or more TKIs and patients with baseline liver metastases. This study showed that, in patients with sensitizing EGFR mutations (e.g., exon 19 deletion or Leu858Arg) and patients with baseline liver metastases, the regimen atezolizumab plus bevacizumab plus carboplatin plus paclitaxel (ABCP) provided overall survival (OS) and PFS benefits compared with the standard-of-care regimen bevacizumab plus carboplatin plus paclitaxel (BCP). Improved OS and PFS obtained by $\mathrm{ABCP}$ versus $\mathrm{BCP}$ was also observed in the intention-to-treat population (3). The results of this study suggest that antiangiogenic agents combined with immunotherapy for patients with EGFRTKI resistance may be an effective treatment.

Our patient was an elderly woman with an NSCLC resistant to first- and third-generation EGFR-TKIs, who was in poor physical condition. She was then administered camrelizumab (SHR-1210), combined with a minimum dose of apatinib (125 mg po qd). After 4 weeks of this regimen, her lung lesions were markedly smaller and necrotic, her breathing problems were markedly reduced, and her physical status was greatly improved. The side effects of this treatment were small and the patient was well tolerated.

The disadvantage of the case is that the patient could not tolerate the puncture biopsy when the lung lesion progresses, and the tissue biopsy and genetic test results of the lung lesion have not been obtained. Although lung lesions progressed significantly, liver metastases did not progress. It cannot be ruled out the existence of tumor heterogeneity. In the future treatment process, it may be necessary to replenish the genetic testing of different tissues according to the specific situation.

\section{Conclusions}

This case report describes a successful attempt to treat an NSCLC patient with EGFR-TKI resistance by a combination therapy consisting of an anti-PD-1 antibody and antiangiogenic agent. This strategy has great potential to overcome EGFR-TKI resistance in NSCLC patients. 
However, before this option can be used as a universal treatment approach, more information from clinical cases administered this treatment should be reported.

\section{Acknowledgments}

We thank Prof. Huawei Zou from Shenging Hospital of China Medical University for the revision of this manuscript. Funding: None.

\section{Footnote}

Reporting Checklist: The authors have completed the CARE Reporting Checklist. Available at http://dx.doi. org/10.21037/apm-19-462

Conflicts of Interest: All authors have completed the ICMJE uniform disclosure form (available at http://dx.doi. org/10.21037/apm-19-462). The authors have no conflicts of interest to declare.

Ethical Statement: The authors are accountable for all aspects of the work and for ensuring that questions related to the accuracy or integrity of any part of the work are appropriately investigated and resolved. All procedures performed in studies involving human participants were in accordance with the ethical standards of the institutional and/or national research committee(s) and with the Helsinki Declaration (as revised in 2013). Written informed consent was obtained from the patient for publication of this manuscript and any accompanying images.

Open Access Statement: This is an Open Access article distributed in accordance with the Creative Commons Attribution-NonCommercial-NoDerivs 4.0 International License (CC BY-NC-ND 4.0), which permits the noncommercial replication and distribution of the article with the strict proviso that no changes or edits are made and the original work is properly cited (including links to both the formal publication through the relevant DOI and the license). See: https://creativecommons.org/licenses/by-nc-nd/4.0/.

\section{References}

1. Mok TS, Wu YL, Ahn MJ, et al. Osimertinib or PlatinumPemetrexed in EGFR T790M-Positive Lung Cancer. N
Engl J Med 2017;376:629-40.

2. Oxnard GR, Hu Y, Mileham KF, et al. Assessment of Resistance Mechanisms and Clinical Implications in Patients With EGFR T790M-Positive Lung Cancer and Acquired Resistance to Osimertinib. JAMA Oncol 2018;4:1527-34.

3. Reck M, Mok TSK, Nishio M, et al. Atezolizumab plus bevacizumab and chemotherapy in non-small-cell lung cancer (IMpower150): key subgroup analyses of patients with EGFR mutations or baseline liver metastases in a randomised, open-label phase 3 trial. Lancet Respir Med 2019;7:387-401.

4. Mo H, Huang J, Xu J, et al. Safety, anti-tumour activity, and pharmacokinetics of fixed-dose SHR-1210, an antiPD-1 antibody in advanced solid tumours: a doseescalation, phase 1 study. Br J Cancer 2018;119:538-45.

5. Li J, Zhao X, Chen L, et al. Safety and pharmacokinetics of novel selective vascular endothelial growth factor receptor-2 inhibitor YN968D1 in patients with advanced malignancies. BMC Cancer 2010;10:529.

6. Ding J, Chen X, Dai X, et al. Simultaneous determination of apatinib and its four major metabolites in human plasma using liquid chromatography-tandem mass spectrometry and its application to a pharmacokinetic study. J Chromatogr B Analyt Technol Biomed Life Sci 2012;895896:108-15.

7. Rami-Porta R, Bolejack V, Crowley J, et al. The IASLC Lung Cancer Staging Project: Proposals for the Revisions of the T Descriptors in the Forthcoming Eighth Edition of the TNM Classification for Lung Cancer. J Thorac Oncol 2015;10:990-1003.

8. Yu HA, Arcila ME, Rekhtman N, et al. Analysis of tumor specimens at the time of acquired resistance to EGFR-TKI therapy in 155 patients with EGFR-mutant lung cancers. Clin Cancer Res 2013;19:2240-7.

9. Lim SM, Syn NL, Cho BC, et al. Acquired resistance to EGFR targeted therapy in non-small cell lung cancer: Mechanisms and therapeutic strategies. Cancer Treat Rev 2018;65:1-10.

Cite this article as: Cong $\mathrm{X}$, Chen J, Zheng W. The combination of camrelizumab and apatinib obtained ongoing partial remission for a patient with osimertinib-resistant non-small cell lung cancer: case report. Ann Palliat Med 2021;10(3):3469-3474. doi: 10.21037/apm-19-462 